\title{
Problemas de gestión asociados al liderazgo como función directiva*
}

\author{
Management problems associated to leadership as a directive function \\ Problemas de gestão associados à liderança como função diretiva
}

\author{
Jorge Ulloa Garrido ${ }^{1}$, Oscar Nail Kröyer ${ }^{2}$, Abelardo Castro Hidalgo ${ }^{3}$, Máximo Muñoz \\ Reyes $^{4}$ \\ ${ }^{1}$ Facultad de Educación, Universidad de Concepción, Tel: 041-2204107, jorulloa@udec.cl \\ ${ }^{2}$ Facultad de Educación, Universidad de Concepción. Tel: 041-2207483, onail@udec.cl \\ ${ }^{3}$ Facultad de Educación, Universidad de Concepción. Tel: 041-2204221, acastro@udec.cl \\ ${ }^{4}$ Facultad de Educación, Universidad de Concepción. Tel: 041-2204107, maximunoz@udec.cl
}

\begin{abstract}
RESUMEN
El presente artículo presenta resultados de un estudio que busca identificar los problemas de gestión que reconocen los Director/as de escuelas municipales urbanas de la provincia de Concepción, y el orden de importancia que asignan a cada uno de ellos. La mayoría de los problemas enunciados son atribuibles a factores y actores externos no asociados directamente al proceso de aprendizaje y la enseñanza en el aula, considerando que las escuelas municipales que atienden a sectores vulnerables no presentan resultados educativos satisfactorios a nivel nacional, lo que se refleja en las mediciones estandarizadas aplicadas por el Ministerio de Educación de Chile.
\end{abstract}

Palabras clave: liderazgo directivo, gestión del conocimiento, mejoramiento educativo, problemas de liderazgo.

\begin{abstract}
This paper presents the results of a study that intends to identify management problems recognized by the Head Teachers of urban municipal schools of the province of Concepción and the order of importance they assign to each of them. The majority of the problems expressed can be attributed to external factors and agents not directly connected with the teaching-learning process in the classroom. It is possible to indicate that municipal schools that serve vulnerable sectors do not display satisfactory educational results at national levels, which is reflected in the standardized measurements applied by Ministry of Education of Chile.
\end{abstract}

Key words: directive leadership, knowledge management, educational improvement, leadership issues.

\section{RESUMO}

Apresentam-se resultados de estudo que objetiva identificar os problemas de gestão reconhecidos por Diretores(as) das escolas municipais urbanas da província de Concepción e a ordem de importância dada a cada um dos problemas identificados. A maioria dos problemas enunciados são atriuídos a fatores e sujeitos externos não associados diretamente ao processo ensino-aprendizagem dos esatudantes, em salas aula. Ressalta-se que as escolas municipais atendem a setores vulneráveis e apresentam resultados insatisfatórios, em nível nacional, o que se reflete nas avaliações externas propostas Ministério da Educação do Chile.

Palavra schave: liderança diretiva, gestão do conhecimento, melhoramento educativo, problemas de liderança.

* Investigación realizada con el financiamiento del Centro de Investigaciones Avanzadas en Educación (CIAE), Universidad de Chile, Universidad Católica de Valparaíso y Universidad de Concepción. Proyecto Fondap 2009-2011. 


\section{REVISIÓN DE LA LITERATURA}

Las investigaciones asociadas a la línea de "escuelas efectivas", plantean que el mejoramiento educativo y éxito escolar de los estudiantes se juega en la sala de clases (Raczynski y Muñoz, 2005; Marcel, 2009). En este sentido Hopkins (2009) ha planteado una trilogía interesante en torno al mejoramiento educativo: Aprendizaje Potente, Enseñanza Potente y Escuela Potente. Es necesario desarrollar en los estudiantes habilidades funcionales, habilidades de pensar y aprender y habilidades personales que les permita desarrollar su efectividad personal y de empleabilidad. La enseñanza y el aprendizaje potente se desenvuelven mejor bajo ciertas condiciones institucionales como el compromiso con el desarrollo docente, involucramiento de todos los agentes en la toma de decisiones, liderazgo de instrucción, planificación colaborativa, coordinaciones efectivas, investigación y reflexión. Estas condiciones constituyen el andamiaje básico que le da sustento al mejoramiento educativo en la institución, especialmente al desarrollo de una enseñanza efectiva para el logro de aprendizajes efectivos. En este sentido, Robinson (2010) plantea que la mejora en los resultados de aprendizaje de los alumnos está fuertemente ligada al liderazgo. Un liderazgo efectivo del Director o Directora, plantea Robinson, se traduce fundamentalmente en el establecimiento de metas y expectativas de aprendizaje compartidas con los docentes, la planificación, coordinación y evaluación de la enseñanza y del currículo y, de mayor impacto, en la promoción y participación en el aprendizaje y desarrollo docente. Esto último está fuertemente asociado a la corriente investigativa de mejoramiento y cambio educativo que ha planteado el desarrollo de comunidades de aprendizaje que permitan la generación de conocimiento educativo e involucren a los docentes y directivos en ese proceso (Day et al., 2009; Fullan, 2002; Hargreaves, 2003; Santos, 2002).

Las investigaciones encabezadas por Leithwood (2009) plantean que el liderazgo es un fenómeno social, implica un propósito y una dirección, es contextual y contingente y es una función. El rol del liderazgo es ejercido fundamentalmente por directores o directoras y profesores o profesoras y tiene un efecto demostrable en el desempeño escolar. De la misma forma, plantea que un conjunto de prácticas son valiosas independientemente del contexto en que el liderazgo se desarrolle. Entre otras, establecer rumbos a través de la identificación y articulación de una visión y el establecimiento de altas expectativas de rendimiento; desarrollar a las personas, rediseñar la organización, especialmente la cultura escolar.

El aumento en los niveles de cobertura del sistema educativo chileno, y con ello la disminución de las brechas educativas entre los distintos grupos socioeconómicos, ha planteado nuevos desafíos. En efecto, tener capacidad de retención de esa población escolar incorporada al sistema, así como el ofrecer una educación de calidad en función de las necesidades de una población diversa, constituyen claramente elementos fuertemente demandados (Ministerio de Educación de Chile, 2006).

En los últimos 20 años de desarrollo educacional chileno, se ha originado un sistema altamente estratificado tanto desde el punto de vista socioeconómico, como desde el punto de vista de los resultados en las mediciones estandarizadas de logros de aprendizaje realizadas (OCDE 2004). En este contexto, desde el punto de vista de los resultados absolutos de las mediciones realizadas a través del Sistema de Medición de la Calidad y Equidad de la Educación en Chile, SIMCE, la educación municipal presenta los resultados 
más bajos. Sin embargo, este sector atiende actualmente al $46 \%$ de la población escolar chilena. De este grupo, alrededor de un $70 \%$ de corresponde a alumnos de los sectores socioeconómicos más bajos, siendo los colegios de dependencia municipal, la principal oferta educativa para ellos. Los estudios realizados en Chile sugieren que al comparar resultados de las mediciones de logros de aprendizaje estandarizadas, los establecimientos de dependencia municipal logran resultados similares que los de dependencia particular (Marcel, 2009). Pareciera que existe acuerdo de que los resultados SIMCE se explican fuertemente por el origen socioeconómico de los estudiantes, más que por la dependencia o tipo de sostenedor de las escuelas (González et al., 2002).

Los resultados de las escuelas municipales, no obstante, mirados respecto de sí mismas, no son satisfactorios. Los estudios de efectividad escolar en sectores de pobreza realizados en Chile, por su parte, ha aportado luces interesantes respecto de las claves del éxito en el proceso de mejoramiento educativo de establecimientos de dependencia municipal. Factores como disponer de equipos de gestión, desarrollo de competencias docentes, informar y reflexionar acerca de los resultados SIMCE (Paredes y Lizama, 2006); una gestión docente centrada en el aprendizaje, que evalúa y retroalimenta el logro de los estudiantes en un ambiente de buena relación alumnos-profesores (Raczynski y Muñoz, 2005; Unicef, 2004), están a la base de las explicaciones de éxito escolar alcanzados por algunas de las instituciones escolares que atienden población escolar de sectores de alta vulnerabilidad.

En este contexto, es relevante seguir profundizando en torno a la forma en que los directores de este tipo de escuelas enfrentan los problemas de gestión, cuáles son los focos de sus preocupaciones y qué hacen para enfrentarlas.

\section{METODOLOGÍA DE LA INVESTIGACIÓN}

La investigación desarrollada ha tenido como objetivo identificar los principales problemas de gestión, asociadas a la función de Liderazgo, reconocidos por los Director/ as de escuelas municipales urbanas de la provincia de Concepción, y la relevancia que le asignan a éstos.

Se trabajó con una población de $\mathrm{N}=121$ directores de establecimientos vulnerables, municipales, básicos y urbanos de la provincia de Concepción. La muestra definida aleatoriamente correspondió a $\mathrm{n}=44$ directores. El 51\% corresponde a hombres y $49 \%$ a mujeres. El 33\% tiene menos de 55 años y el $67 \%$ tiene más de 56 años de edad. El $44 \%$ tiene cinco o menos años de experiencia en el cargo y $22 \%$ entre 6 y 10 años. El $11 \%$ tiene más de 31 años de experiencia.

Se aplicó a los sujetos participantes de la investigación un cuestionario consistente en 43 problemas. El cuestionario contiene cuatro tipos de variables: a) socio-demográficas de identificación del encuestado, b) de incidencia de problemas relativos la presencia/ ausencia de cada uno de estos c) variables discretas de intensidad de los problemas en escala de 1 a 10 y d) variables de soluciones propuestas

A su vez, se aplicaron dos pruebas estadísticas para la validación interna del cuestionario: Análisis Factorial y Alfa de Crombach:

El Análisis factorial se configuró con el fin de deducir y verificar las dimensiones o categorías para los problemas identificados en base a sus intensidades. Estas categorías 
se detallan en la Tabla 1. El análisis de componentes principales revela que con 2 grupos de variables (preguntas del instrumento), se explica un 100\% de la información, y el coeficiente de correlación cofenética es de 1 . El primer componente explica un $74 \%$ y el segundo un $26 \%$.

Por otro lado el Alfa de Crombach se aplicó para determinar la consistencia interna del cuestionario. Este estadístico arrojó que la itemización es adecuada, ya que revela buena asociación entre los ítems del área de gestión:

\begin{tabular}{|c|c|c|}
\hline Área de Gestión & Alfa de Crombach & \multirow{2}{*}{ Global } \\
\hline Liderazgo & 0,750 & \multirow{2}{*}{0,945} \\
\hline Gestión Curricular & 0,794 & \\
\hline Recursos & 0,861 & \\
\hline $\begin{array}{c}\text { Clima Organizacional y } \\
\text { Convivencia }\end{array}$ & 0,860 & \\
\hline
\end{tabular}

Tabla 1. Estadísticos Alfa de Crombach por Área de Gestión

El estadístico Alfa de Crombach global asume un valor de $\mathbb{\nabla}=0,945$, de donde se concluye que el cuestionario es altamente consistente.

Los 43 problemas incluidos en el cuestionario fueron agrupados en cuatro áreas de gestión del Sistema de Aseguramiento de la Calidad de la Gestión, SACGE, Ministerio de Educación de Chile, y sub-categorizados por el equipo de especialistas investigadores de las áreas de Gestión Escolar y de Ciencias Económicas y Administrativas. Las cuatro categorías son: Liderazgo, Gestión Curricular, Recursos, Clima Organizacional y Convivencia. Cada una de estas categorías está subdivida en sub-categorías específicas. Para fines de esta investigación, mencionaremos solo las sub-categorías pertenecientes al área de Liderazgo:

\begin{tabular}{|c|c|c|}
\hline Categoría & Sub-Categoría & $\mathbf{N}^{\mathbf{0}}$ Problema \\
\hline \multirow{3}{*}{ Liderazgo } & Tiempo & 1,2 y 3 \\
\cline { 2 - 3 } & Autonomía & 4,5 y 6 \\
\cline { 2 - 3 } & Planificación Institucional & 7,8 y 9 \\
\hline
\end{tabular}

Tabla 2. Sub-categorías área Liderazgo.

Los datos fueron analizados de manera descriptiva, a saber, se ordenaron los problemas de esta categoría por nivel de intensidad para su posterior análisis, mostrando la posible vinculación que existe entre algunos problemas y sus causas posibles. 


\section{INTENSIDAD DE LOS PROBLEMAS ASOCIADOS A LA DIMENSIÓN DE LIDERAZGO}

\begin{tabular}{|c|c|c|}
\hline $\begin{array}{c}\mathbf{N}^{\mathbf{0}} \\
\text { Problema }\end{array}$ & Problema & Intensidad \\
\hline 4 & $\begin{array}{c}\text { Ausencia de facultades y/o atribuciones para la selección, } \\
\text { contratación y despido de personal. }\end{array}$ & 7,74 \\
\hline 1 & Cómo utilizar el tiempo en las distintas áreas de gestión & 6,95 \\
\hline 5 & Ausencia de facultades y/o atribuciones para administrar recursos \\
financieros & 6,86 \\
\hline 3 & Mucho tiempo dedicado a tareas ajenas a la gestión pedagógico- \\
curricular & 6,59 \\
\hline 2 & Carencia de tiempo para las distintas tareas de gestión & 6,56 \\
\hline 6 & Ausencia de facultades y/o atribuciones para administrar recursos & 4,42 \\
\hline 9 & materiales & 3,61 \\
\hline 7 & Falta de competencias en equipo directivo para la planificación & 3,45 \\
\hline 8 & Dificultades para contar con un PEI actualizado y pertinente a la \\
realidad del establecimiento educativo & 2,98 \\
\hline
\end{tabular}

Tabla 3. Problemas de Liderazgo en orden descendente según intensidad.

El cuadro indica que el problema "Ausencia de facultades y/o atribuciones para la selección, contratación y despido de personal" es el que tiene mayor intensidad promedio: 7,74. Este problema está relacionado con el funcionamiento del sistema educacional chileno, en el cual los establecimientos públicos, dependientes de las municipalidades, no cuentan con la potestad para gestionar el recurso humano que tienen a disposición. La gestión de éste está en manos de los Departamentos de Educación Municipal (DEM) de cada comuna, al cual los directores de las escuelas deben recurrir en cada ocasión que se requiera hacer modificaciones a la planta docente y/o administrativa. La importancia de este problema radica, además, en el efecto que tiene en el proceso de aprendizaje de los estudiantes y en el cumplimiento de las metas institucionales, ya que esta incapacidad directiva para administrar los recursos humanos impide una pronta respuesta ante fenómenos en los cuales se produce una ausencia de docentes por distintos motivos. Se debe agregar que este problema solo se centra en la ausencia de facultades, pero no hace referencia a cómo los directores motivan o potencian la labor docente al interior de los establecimientos, es decir, se refiere solo a una dimensión de la gestión del recurso humano.

Este problema se vincula con el problema $\mathrm{N}^{\mathrm{o}} 5$ "Ausencia de facultades y/o atribuciones para administrar recursos financieros" el cual presenta la necesidad del director de recurrir a los departamentos de educación municipal para solicitar recursos financieros cuando sea necesario. Nuevamente aparece la dependencia director-sostenedor como un problema 
importante para los directores, ya que limita su capacidad de gestión y de innovación (compra de materiales, reposición de elementos antiguos o en mal estado, introducción de nuevas metodologías, contratación de personal de apoyo a la labor docente, etc) en los establecimientos y, por tanto, tendría un impacto negativo en los procesos de enseñanza del alumnado y en la mejora de la calidad educativa de las escuelas.

Relacionado con estos dos problemas, aunque con menor intensidad para los directores, se observa el problema $\mathrm{N}^{\circ}$ 6: "Ausencia de facultades y/o atribuciones para administrar recursos materiales", con una intensidad promedio de 4,42. La asignación de una importancia significativamente menor a los dos problemas antes mencionados, a pesar de la relación existente entre estos, puede tener entre sus causas la existencia de material suficiente para desarrollar la labor pedagógica y extra-pedagógica tanto dentro como fuera del aula, por lo cual la gestión directiva en este tema no tendría mayores niveles de conflicto. La segunda causa posible hace referencia el problema anterior, entendiéndose que teniendo facultades para administrar recursos financieros se tendrán las facultades para administrar recursos materiales. En otras palabras, se podría postular que los directores realizaron una vinculación entre recursos financieros y recursos materiales, siendo prioritaria la gestión de recursos financieros para gestionar recursos materiales. La gestión de materiales estaría supeditada a la gestión de dinero o sería consecuencia de ésta.

El problema: utilizar el tiempo en las distintas áreas de gestión tiene una intensidad promedio de 6,95. Esta dificultad ocupa el segundo lugar de importancia para los directores y se articula en torno a la planificación que hacen los directivos del tiempo institucional y su utilización en las áreas de gestión y las labores que competen a cada una de estas. Este problema de no saber cómo utilizar u organizar el tiempo se traduce en efectos concretos como el abandono de aspectos de la gestión institucional y su posterior efecto en el desarrollo de las escuelas, además de impactar en el proceso de aprendizaje de los alumnos.

Mucho tiempo dedicado a tareas ajenas a la gestión pedagógico-curricular ocupa el cuarto lugar con una intensidad promedio de 6,59. Esta dificultad puede ser interpretada desde dos dimensiones: por un lado, asociada con el problema anterior, no saber cómo utilizar el tiempo produce un desaprovechamiento de éste en tareas ajenas o con poca relación. El enfoque directivo cambia desde sus funciones de gestión hacia algunas que no necesariamente son de su competencia o prioritarias para su cargo. La otra dimensión desde la cual se puede interpretar este problema indica que dada la multiplicidad de tareas que se presentan en los establecimientos educacionales, el director debe asumir por necesidad y/u obligación labores que están circunscritas a su competencia, destinando tiempo a estas y restando tiempo a los procesos de gestión. La causa de esto puede ser la escasez de personal, personal con pocas competencias y/o un liderazgo demasiado personalista y autoritario que limita la delegación de tareas a los subalternos o posibles conflictos de rol en los cuales no están claros los distintos "papeles" que cada sujeto cumple al interior de las organizaciones y las tareas que competen a cada uno.

Carencia de tiempo para las distintas tareas de gestión ocupa el quinto lugar con una intensidad promedio de 6,56. La diferencia entre este problema y el presentado anteriormente es marginal: 0,03 y es posible analizarlo desde la perspectiva ya presentada. El no saber cómo utilizar el tiempo provoca que se destine parte de éste a otras tareas, lo cual finalmente tiene como consecuencia última una carencia de tiempo para desarrollar las tareas de la gestión institucional. Además se puede agregar la perspectiva 
de la ausencia de personal, la carencia de personal calificado y/o el estilo de liderazgo de los directores. Se observa en los tres problemas vinculados al tiempo dedicado a la gestión una diferencia marginal entre las intensidades, lo cual demostraría la vinculación existente entre estas dificultades y la alta importancia que poseen para los directores.

Para concluir y con una importancia significativamente menor a los problemas antes descritos, se encuentra: Cultura institucional que no favorece la planificación institucional con una intensidad promedio de 3,61. Este problema a diferencia de los demás hace referencia a procesos que subyacen a los integrantes de los establecimientos y de los cuales estos no son conscientes y/o no se percatan y que exceden, la mayoría de las veces, la acción de los directores en sus roles de líder. La cultura de una organización, en este caso de establecimientos educativos, está configurada por una multiplicidad de elementos que hacen lenta y dificultosa su modificación. De esta manera, la cultura puede ser entendida como un proceso en el cual los cambios presentes, ya sea en el orden de las decisiones como en el plano de la interacción entre los miembros y los símbolos y códigos organizacionales, pueden tener un efecto en el mediano y largo plazo. El bajo nivel de importancia que los directores asignan a este problema puede deberse a tres posibles factores: que efectivamente existe una cultura institucional que favorezca la planificación institucional en la mayoría de los establecimientos; un segundo factor posible es la poca importancia asignada a alguna de las variables presentadas (cultura de la organización y/o planificación) y, finalmente, un tercer factor posible es que los directores consideren que si bien el problema está presente en la mayoría de los establecimientos, éste no causa grandes efectos y, por consecuencia, su nivel de importancia es considerada menor.

El problema Falta de competencias en equipo directivo para la planificación institucional (PEI, Planes de Mejora, etc.) con una intensidad promedio de 3,45 ocupa el penúltimo lugar, y es calificado de baja importancia por los directores. Esto permite postular que este problema no genera mayor impacto en los establecimientos educacionales y/o se cuenta con el personal calificado para realizar la planificación institucional. Este problema, además, involucra la capacidad de los directores de conducir y motivar al personal que tiene bajo su cargo para la mejora constante de los establecimientos. Hace referencia de manera indirecta al rol de líder que cumple cada director en su establecimiento y a su capacidad de congregar y motivar a las personas bajo su cargo para un proyecto en común.

Para concluir, el último problema señalado: Dificultades para contar con un PEI (Proyecto Educativo Institucional) actualizado y pertinente a la realidad del establecimiento educativo, tiene asignado una intensidad promedio 2,98. Este problema es el de menor importancia de todos los presentados, ya sea porque el PEI esta correctamente formulado en los establecimientos, porque se realizan constantes revisiones y modificaciones a éste y/o porque pesar de la importancia fundamental de este documento al interior de las escuelas, se minimiza el efecto negativo que tiene una discordancia entre PEI-establecimiento por parte de los directores, en comparación a otros aspectos de la gestión.

\section{CONCLUSIONES}

La investigación en esta primera etapa se propuso identificar los problemas de mayor intensidad que reconocen enfrentar los Directores/as de Escuelas Básicas Municipales que atienden población escolar de sectores vulnerables, asociados a la función de liderazgo. 
Claramente, los Directores se preocupan de variables externas a los procesos de enseñanza aprendizajes de sus alumnos y les atribuyen a éstas mayor importancia en los procesos de gestión. En efecto, reconocen como problemas de mayor relevancia la "Ausencia de facultades y/o atribuciones para la selección, contratación y despido de personal"; "Ausencia de facultades y/o atribuciones para administrar recursos financieros"; Carencia de tiempo para las distintas tareas de gestión"; Ausencia de facultades y/o atribuciones para administrar recursos materiales". Los problemas se originan en otro nivel del sistema y, por tanto, las soluciones están allí también. Es una visión inmovilizadora de la gestión y el ejercicio del Liderazgo. Esto actúa como una suerte de mecanismo de defensa ante los problemas de la escuela. Tendríamos que preguntarnos, ¿qué estamos haciendo a nivel local (Municipio) o a nivel central (Ministerio de Educación de Chile) para que estos Directores sean más autónomos y emprendedores respecto a sus propios establecimientos? ¿es posible, en este panorama, hablar de gestión directiva? ¿es posible hablar de liderazgo escolar?

Otra variable que es transversal a los problemas planteados es la falta de tiempo de la escuela para realizar lo pedagógico como su foco prioritario; ocurre que, por falta de tiempo o por mala administración de éste, los tiempos lectivos o clases efectivas, con el trabajo pedagógico de los profesores, quedan relegados, argumentándose "falta de tiempo", lo que impide que se visualicen otros problemas que tienen que ver con los "procesos de cambio" al interior de las practicas pedagógicas. Al parecer los directores siguen mirando la escuela desde afuera.

Otra dificultad que se constata es la dificultad de los directores para conformar un PEI en los establecimientos. Si bien se reconoce la baja intensidad de este elemento, se debe destacar que este problema vincula de manera directa el rol de líder que deben cumplir los directores en las escuelas. Se trata de gestionar para consensuar en la comunidad educativa un proyecto en común sobre qué quieren de las escuelas, qué competencias serán prioritarias, cuál es la visión y la misión de los establecimientos. Por lo tanto, la presencia de este problema, más allá de la importancia asignada revela las debilidades directivas en cumplir efectivamente el rol de líder par guiar a la escuela en este proceso de conducir un claro plan estratégico de desarrollo de la escuela que queremos.

También llama la atención que se indique como problemas la falta de competencia de los equipos directivos, que acompañan en la labor de gestión al Director. ¿Qué pasa con los equipos de gestión de los establecimientos, quién los elige, qué perfil tienen éstos, quién determina su competencia? Ya es sabida la importancia que tienen los equipos de trabajo para llevar a una escuela a ser efectiva, ¿por qué entonces la administración municipal no ha sabido conformar estos equipos competentes y profesionales, cómo enfrentar los desafíos de la escuela sin equipos competentes claves en la organización de una escuela?

No es posible reconocer el aprendizaje de los estudiantes y el proceso de enseñanza como eje central de sus preocupaciones, no obstante, los resultados de aprendizaje de este tipo de instituciones educativas demuestran que éste es un foco crítico. Las investigaciones asociadas a la efectividad escolar y mejoramiento educativo han demostrado que los directores de este tipo de escuelas tienen una mirada opuesta. Las preocupaciones son esencialmente pedagógico curriculares y asumen las responsabilidades de ello.

Esta mirada de la gestión educativa y este tipo de liderazgo que desarrollan los directores en escuelas municipales nos plantea nuevas interrogantes. ¿Comprenden la esencia del proceso educativo? ¿los profesores no les permiten entrar al aula para mantener 
independencia? Éste constituye un problema central a dilucidar a fin de proporcionar elementos de política en esta línea.

\section{REFERENCIAS BIBLIOGRÁFICAS}

Day, C., Sammons, P., Hopkins, D., Harris, A., Leithwood, K., Gu, Q., Brown, E., Ahtaridou, E. y Kington, A. (2009). The impact of school leadership: Final Report. Departament for Children, Schools and Families: University of Nottingham.

Fischman, D (2009). Cuando el Liderazgo no es suficiente. Santiago de Chile: Aguilar.

Fullan, M. (2002). El significado del Cambio educativo, un cuarto de siglo de aprendizaje. Revista de Currículum y Formación del Profesorado, n. 6, 1-2.

Gónzalez, P., Mizala, A. y Romanguera, P. (2002). Recursos diferenciados a la educación subvencionada en Chile. Santiago de Chile: Universidad de Chile.

Hopkins, D. (2009). Mi Escuela Una Gran Escuela. Santiago de Chile: LOM.

Hargreaves, A (2003). Enseñar en la sociedad del Conocimiento. Barcelona: Ocraedro.

Leithwood, K, (2009). ¿Cómo liderar nuestras escuelas? Aportes desde la investigación. Santiago de Chile: Salesianos Impresores.

Marcel, M. (2009). ¿Porqué la Educación Municipal?. En M, Marcel. \& D, Raczynski (Eds.), La asignatura pendiente: Claves para la revalidación de la educación pública de gestión local en Chile (pp. 33-39). Santiago de Chile: Uqbar.

MINISTERIO DE EDUCACIÓN DE CHILE (2006). Informe final concejo asesor presidencial para la calidad de la educación. Santiago de Chile: Autor.

OCDE (2004). Revisión de Políticas Nacionales de Educación: Chile. París: Ministerio de Educación de Chile.

Paredes, R. y Lizama, O. (2006). Restricciones, Gestión y Brecha Educativa en Escuelas Municipales. Documento Centro de Políticas Públicas. Santiago de Chile: Pontificia Universidad Católica.

Raczynski, D. \& Muñoz, G. (2005). Efectividad escolar y cambio educativo en condiciones de pobreza en Chile. Santiago de Chile: Ministerio de Educación de Chile.

Robinson, V. (2010). Del Liderazgo al resultado de los estudiantes. Obtenido el 26 de Marzo de 2010 de http://www.educarchile.cl

Santos, M (2002). La Escuela que aprende. Madrid: Morata.

UNICEF (2004). ¿Quien dijo que no se puede?. Escuelas efectivas en sectores de pobreza. Santiago de Chile: Unicef, Mineduc. 
